\title{
Cues for Directional Hearing in the Fly Ormia ochracea
}

\author{
Andrew C. Mason* \\ Integrative Behaviour and Neuroscience Group, Department of Biological Sciences, University of Toronto Scarborough, \\ Toronto, ON, Canada
}

Insects are often small relative to the wavelengths of sounds they need to localize, which presents a fundamental biophysical problem. Understanding novel solutions to this limitation can provide insights for biomimetic technologies. Such an approach has been successful using the fly Ormia ochracea (Diptera: Tachinidae) as a model. O. ochracea is a parasitoid species whose larvae develop as internal parasites within crickets (Gryllidae). In nature, female flies find singing male crickets by phonotaxis, despite severe constraints on directional hearing due to their small size. A physical coupling between the two tympanal membranes allows the flies to obtain information about sound source direction with high accuracy because it generates interaural time-differences (ITD) and interaural level differences (ILD) in tympanal vibrations that are exaggerated relative to the small arrival-time difference at the two ears, that is the only cue available in the sound

OPEN ACCESS

Edited by:

Fernando Montealegre-Z, University of Lincoln, United Kingdom

Reviewed by: Heiner Römer,

University of Graz, Austria Bernhard Ronacher, Humboldt University of Berlin, Germany

*Correspondence:

Andrew C. Mason andrew.mason@utoronto.ca

Specialty section:

This article was submitted to Behavioral and Evolutionary Ecology,

a section of the journal

Frontiers in Ecology and Evolution

Received: 10 March 2021

Accepted: 04 June 2021

Published: 02 July 2021

Citation:

Mason AC (2021) Cues for Directional Hearing in the Fly Ormia ochracea.

Front. Ecol. Evol. 9:679064. doi: 10.3389/fevo.2021.679064 stimulus. In this study, I demonstrate that pure time-differences in the neural responses to sound stimuli are sufficient for auditory directionality in $O$. ochracea.

Keywords: directional hearing, eardrum, insect, phonotaxis, interaural difference, coupled ears, Ormia

\section{INTRODUCTION}

The fly Ormia ochracea (Diptera:Tachinidae) possesses an auditory system that performs analagous functions to those of vertebrate hearing (detection, recognition, segregation, and localization or sources), albeit for a restricted range of stimuli (Gray et al., 2007). The flies are parasitoids and females must locate a cricket host in order to reproduce (Wineriter and Walker, 1990). They accomplish this by localizing the calls of singing male crickets using an auditory system dedicated to this task (Cade, 1975). Tympanal hearing is unusual for flies. All known examples are species that are parasitoids of acoustic insects (Allen, 1995; Robert et al., 1999; Lehmann, 2003), and these include two families (Tachinidae and Sarcophagidae) in which tympanal hearing has evolved independently through convergent adaptation of the same precursor organ (Edgecomb et al., 1995; Robert et al., 1996a; Lakes-Harlan et al., 1999).

Due to the small size of the flies (ears are $<0.5 \mathrm{~mm}$ apart) relative to the wavelength of cricket sound $(\sim 7 \mathrm{~cm})$, acoustic directional cues are severely restricted (Kuhn, 1987). Sound waves impinging on the fly auditory system generate no interaural level difference (ILD) and interaural time differences (ITDs) are very small (maximum $1.5 \mu \mathrm{s}$ for a sound source at $90^{\circ}$ relative to the midline axis). Nevertheless, flies can localize a cricket sound source with exceptional accuracy $\left(<2^{\circ}\right.$ azimuth, Mason et al., 2001).

Abbreviations: BMAA, biomimetic antenna array; ICE, internally coupled ears; ILD/ITD, interaural level/time difference; MEMS, micro-electromechanical system; nITD/nILD, neural interaural level/time difference; tITD/tILD, tympanal interaural level/time difference. 
Directional hearing in Ormia is derived from a specialized mechanical coupling between the two tympanal membranes (Robert et al., 1998). Mechanical coupling of the two eardrums amplifies the small direction-dependent ITDs in the sound field, and generates ILDs in the tympanal vibration responses, so that both cues are present in the tympanal (Robert et al., 1996b) and neural responses (Mason et al., 2001; Oshinsky and Hoy, 2002). Modeling of Ormia auditory mechanics (Miles et al., 1995) demonstrated that intertympanal coupling results in a system with two resonant modes of vibration in response to acoustic stimulation (Figure 1): a symmetric mode, in which the two tympani vibrate with equal amplitude and phase; and an antisymmetric mode, in which the two tympani vibrate with equal amplitude but opposite phase. Under normal acoustic conditions, a sound source located directly ahead of the fly $\left(0^{\circ}\right.$ azimuth) generates vibration in the symmetric mode (each tympanum driven by identical sound pressure waves). Sound impinging from any other direction, however, will stimulate a combination of both modes of vibration with the result that the two tympani will respond with different (directiondependent) amplitudes and phases of vibration, with maximum interaural differences of $\sim 12 \mathrm{~dB}$ in amplitude and $\sim 50 \mu$ s delay (Robert et al., 1996b).

Analyses of the mechanical properties of Ormia tympanal membranes (Miles et al., 1995; Robert et al., 1996b; Akçakaya and Nehorai, 2008) have demonstrated that the mechanical coupling between the two eardrums enhances the system's sensitivity to the minute direction-dependent differences in arrival time of sound at the two ears. The nature of this effect is two-fold. (1) The arrival-time difference is amplified to result in a larger ipsilateral-leading phase difference between vibrations of the two tympani, creating a tympanal interaural time difference (tITD). (2) The amplitude of contralateral tympanal vibration is reduced relative to ipsilateral, creating a tympanal interaural level difference (tILD).

The majority of auditory receptors associated with each ear respond with tonic bursts at the onset of sound pulses (Oshinsky and Hoy, 2002) with response latencies that are dependent on tympanal vibration level, such that tILDs result in directiondependent interaural latency differences in receptor responses. These neural interaural time differences (nITD) scale with the azimuth of the sound source location (Mason et al., 2001; Figure 2). Receptor thresholds vary, however, and another effect of tILDs is (direction dependent) differential recruitment of receptors in the two ears, such that directional sound sources will also generate interaural differences in the amplitude of summed neural responses - neural interaural level differences (nILD).

The directional mechanism of interaural coupling in ormiine ears was at first considered to be a unique evolutionary innovation. However, Ormia directional hearing is now considered to be a specialized example of a taxonomically widespread phenomenon by which acoustic directional cues (mainly ITDs) are amplified via interactions between the two ears to generate larger ITDs and ILDs in tympanal vibration which can then be used to encode directional information in neural responses. Internally coupled ears (ICE) include the majority of vertebrate auditory systems (van Hemmen

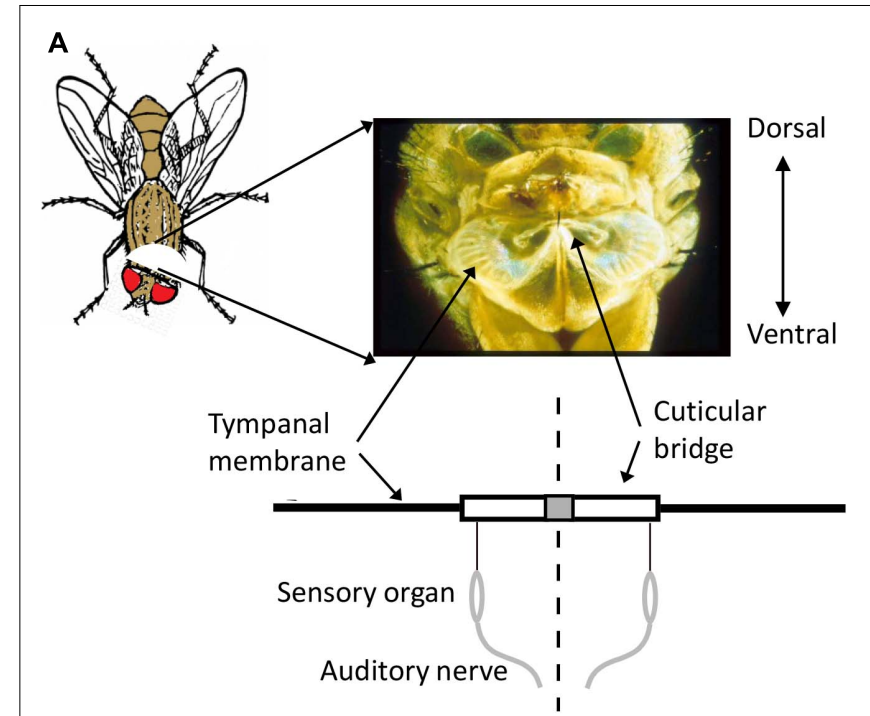

B

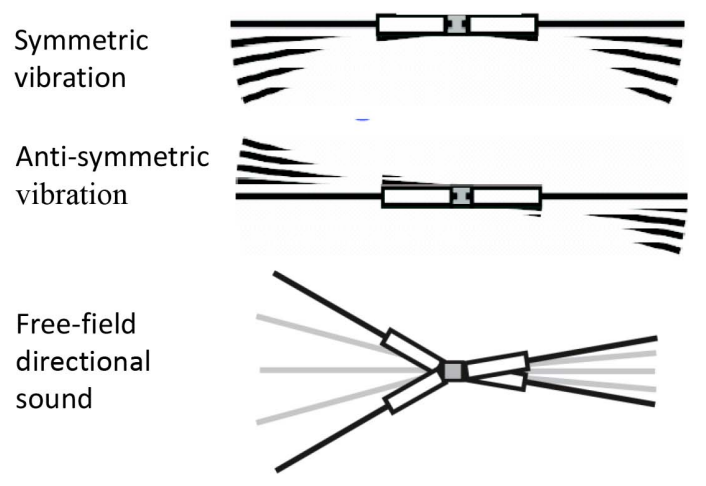

FIGURE 1 | (A) Ormia auditory system. Ears are located behind the head less than $0.5 \mathrm{~mm}$ apart. Mechanical coupling between eardrums, via the cuticular bridge, allows resolution of miniscule interaural time differences - the only acoustic directional cue available. Auditory receptors in each ear are attached to a single point at the end of the cuticular bridge. (B) Symmetric and antisymmetric modes of vibration. Symmetric mode (upper) corresponds to non-directional sound source ( $0^{\circ}$ azimuth). Equal, in-phase sound pressure drives equal-amplitude, in-phase vibration of the two tympani, bending at the midline. In antisymmetric mode vibration (middle) the tympani vibrate with equal amplitudes and opposite phase - rocking like a teeter-totter. Sound stimulation from any direction other than $0^{\circ}$ would elicit tympanal vibration representing a combination of these two modes resulting in unequal vibration of the two tympani (lower).

et al., 2016), and general models of ICE have been derived (Vedurmudi et al., 2016a).

The principle of directionality via coupled hearing has been the basis of multiple auditory adaptations (Römer and Schmidt, 2016), just as insect ears in general show a striking diversity of independent evolutionary origins (Yack and Dawson, 2008). Ormia hearing in particular has been a subject of considerable interest as a model for evolutionary arms races between this eavesdropping parasitoid and its acoustically communicating hosts (e.g., Zuk et al., 1995; Wagner and Basolo, 2007; Sakaguchi and Gray, 2011). 


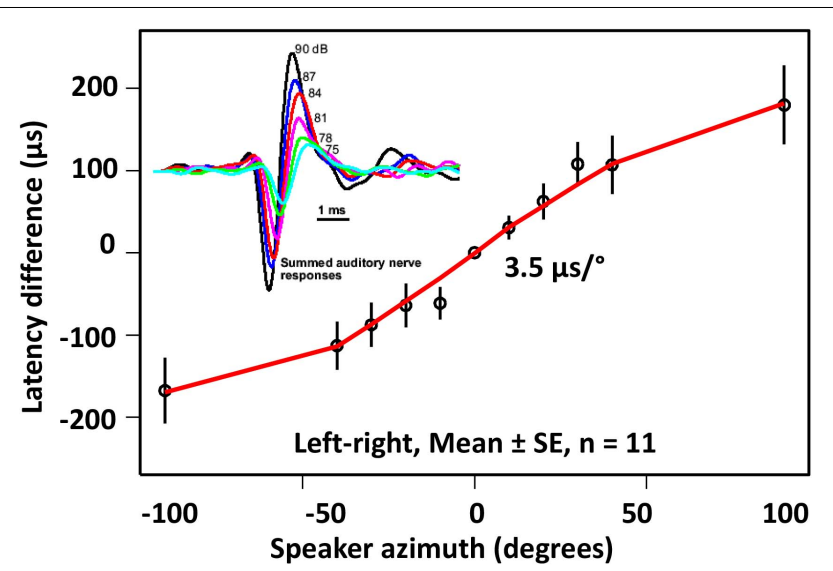

FIGURE 2 | Interaural latency differences as a function of sound source azimuth [redrawn from Mason et al. (2001)]. Inset: Summed auditory nerve responses recorded for different sound levels. Using sound level as a proxy for directional variation in relative tympanal vibration amplitude, these data show that in addition to the nITDs represented in the main plot, contralateral neural responses are weaker for a directional sound source.

Ormia auditory directionality has also emerged as an adaptable model for novel technology, and the past couple of decades have seen considerable interest in biomimetic applications of the flies' intertympanal coupling principle to engineering problems related to source localization for waveform signals, with two main areas of research. Efforts to design biomimetic directional microphones (BDMs, with application, for example, in hearing aids) have sought to mimic the mechanical properties of the fly eardrums in micro-electromechanical-system (MEMS) devices (Ishfaque and Kim, 2018; Zhang et al., 2018). Efforts to design biomimetic antenna arrays (BMAAs) have applied the principle of coupled detectors to electromagnetic signal localization (Grüner et al., 2019). Much of this work has focused on quantifying the contribution of sensor coupling to directional resolution (Akçakaya and Nehorai, 2008; Grüner et al., 2019), tuning the effective bandwidth of the system (Rahaman and Kim, 2020), extending the mechanism to more than one axis (Lisiewski et al., 2011), and optimizing noise-robustness and sensitivity in the readout of ILDs and ITDs (Miles et al., 2009).

Previous work has shown that directional information is represented in the fly auditory system by amplified ITDs in the responses of auditory receptors (Mason et al., 2001). Other work (Oshinsky and Hoy, 2002) suggests interaural differences in the amplitude of neural responses based on differential receptor recruitment may also play a role in directional hearing. There is also evidence that small time-differences between competing auditory sources may play a role in source segregation in Ormia (Lee et al., 2009), and that noise sources may introduce a systematic bias in directional sensing that is a direct consequence of the coupling mechanism (Lee and Mason, 2017). Thus, as in vertebrate hearing, ITDs and ILDs may both contribute to directional hearing in Ormia ochracea, although the way these cues are combined in fly directional hearing is not fully resolved.
This study examines in more detail the relationship between ITDs and ILDs at the tympanal and neural levels, and the contribution of these cues to directional hearing using a series of experiments that measure behavioral, tympanal and neural responses to stimuli with manipulated phase and amplitude to generate ITDs in isolation. The aim is to answer a fairly simple and specific question - can the flies make use of pure time differences in auditory responses to generate directional responses to acoustic stimuli? The results show that interaural time differences do mediate directional responses in the absence of interaural level differences. I consider implications of these data for how ITD and ILD mechanisms may represent alternative solutions to differing physical and evolutionary constraints.

\section{MATERIALS AND METHODS}

\section{Animals}

Experiments were conducted on lab-reared gravid female Ormia ochracea derived from specimens originally collected in Gainesville FL. Flies were maintained at $25^{\circ} \mathrm{C}$ and $75 \%$ humidity on a 12-h:12-h light:dark regime and fed nectar solution (The Birding Company, Yarmouth, MA, United States) ad libitum.

\section{Acoustic Stimuli}

Single tone pulses $(5 \mathrm{kHz}, 10 \mathrm{~ms}$ duration, 0.1 or $0.5 \mathrm{~ms}$ rise/fall time) or synthetic cricket chirps (10 pulses at 50/s) were delivered from two speakers at $84 \mathrm{~dB}$ SPL (unless otherwise specified). Acoustic stimuli were synthesized using Tucker-Davis Technologies (TDT) hardware (System 3) and custom scripts written in $\mathrm{C}$ or Matlab. The stimuli were amplified (NAD S300), passed through a programmable attenuator (TDT model PA5) and broadcast from piezoelectric horn tweeters (Radio Shack Realistic, Taiwan). Stimulus amplitude and timing were controlled by computer and calibrated with a probe microphone (B\&K Type 4182, Denmark). The relative phase and amplitude of simultaneous stimuli were adjusted to manipulate auditory ITDs and ILDs independently (see below).

\section{Experimental Measurements Behavior}

Phonotactic responses were recorded with flies mounted on a spherical treadmill which transduced walking movements for recording by computer (Mason et al., 2001). This openloop setup allowed stimulus conditions to be held constant throughout the duration of presentation. For comparisons of flies walking direction under different stimulus conditions I measured the angle of the fly's trajectory at the halfway point of each (virtual) walking path.

\section{Tympanal Vibration}

Following behavioral experiments, flies' heads were removed and tympanal vibration measured under identical acoustic conditions, using a laser Doppler vibrometer (LDV) (Polytec OFV 3001 controller, OFV 511 sensor head). 


\section{Auditory Nerve Recording}

For some stimulus conditions, I recorded summed auditory nerve responses simultaneously from both ears, under stimulus conditions similar to behavioral and tympanal measurements, using tungsten wire electrodes (AM Systems, $0.25 \mathrm{~mm}$ ). Amplified (AM Systems Model 1800) neural responses were averaged (50 sweeps) and recorded by computer (TDT AD1, $100 \mathrm{kHz}$ sampling rate).

Behavioral, physiological, and mechanical measurements were all carried out in the same setup, with behavioral and mechanical measurements made on the same individuals. Physiological recordings were made on separate cohort of specimens under identical conditions.

I first repeated the measurement of eardrum responses to directional stimuli using the same setup as the other experiments and confirmed comparable results to those in the literature. I then conducted a set of experiments (1-3) aimed a manipulating nITDs and nILDs separately, to address the question of how much each of these response parameters contributes to the coding of auditory directionality.

\section{RESULTS}

\section{Auditory Cues for Sound Localization}

Figure 2 shows variation in the timing and amplitude of summed auditory nerve responses over a $15 \mathrm{~dB}$ range of stimulus levels comparable to the range of tILDs. Previous studies (Oshinsky and Hoy, 2002) have suggested that these nILDs could contribute to the coding of sound source direction, with some data suggesting that nILDs provide more accurate directional information than nITDs (Pollack and Mason, 2014).

I conducted a set of experiments aimed a manipulating nITDs and nILDs separately, to address the question of how each of these response parameters contributes to the coding of auditory directionality.

\section{Experiment 1 - Standing Wave}

This experiment was designed to exploit the antisymmetric mode of tympanal vibration by placing a fly at the node of an acoustic standing wave.

Flies were tethered in place atop the spherical treadmill (Mason et al., 2001), between two speakers positioned at $\pm 90^{\circ}$. Stimuli were calibrated with a probe microphone just above the midline of the tympanal membranes, and the amplitude and timing of identical, but opposite-phase, acoustic stimuli from each speaker were adjusted to create a null at the fly's midline. With a node positioned at the midline of the fly (i.e., the junction of the two tympani), sound pressure acting on the two tympana is equal-amplitude and opposite phase, and the tympanal membranes should rock in the antisymmetric mode of vibration (Figure 1). Reversing the phase of the standing wave would reverse the relative phases of tympanal vibration so that if this phase difference provided a directional cue, flies directional response should also change.

By broadcasting the same stimuli in phase from both speakers, I could also generate a summed waveform at the midline of the fly.

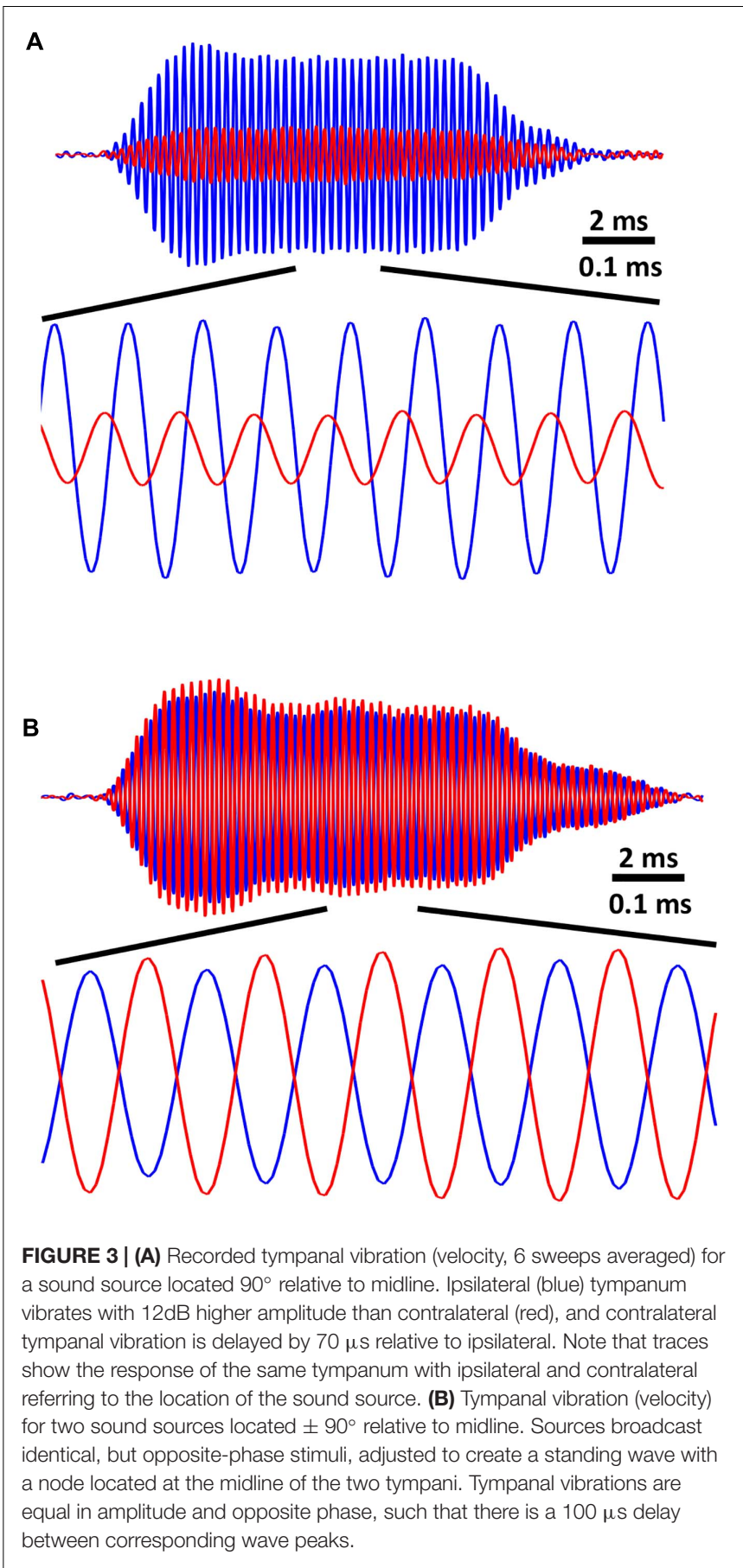

This condition should elicit symmetric mode tympanal vibration (mimicking a phantom source at $0^{\circ}$ azimuth, Lee et al., 2009).

Flies' behavioral responses (phonotaxis toward synthetic cricket chirps) were recorded $(n=6)$ for: (i) directional signals from each speaker individually; (ii) the standing wave condition (signals canceling at the fly's midline), recorded for both relative phases of tympanal vibration (i.e., left-leading and right-leading); and (iii) the summing signals condition. After behavioral recordings I measured tympanal vibration under 


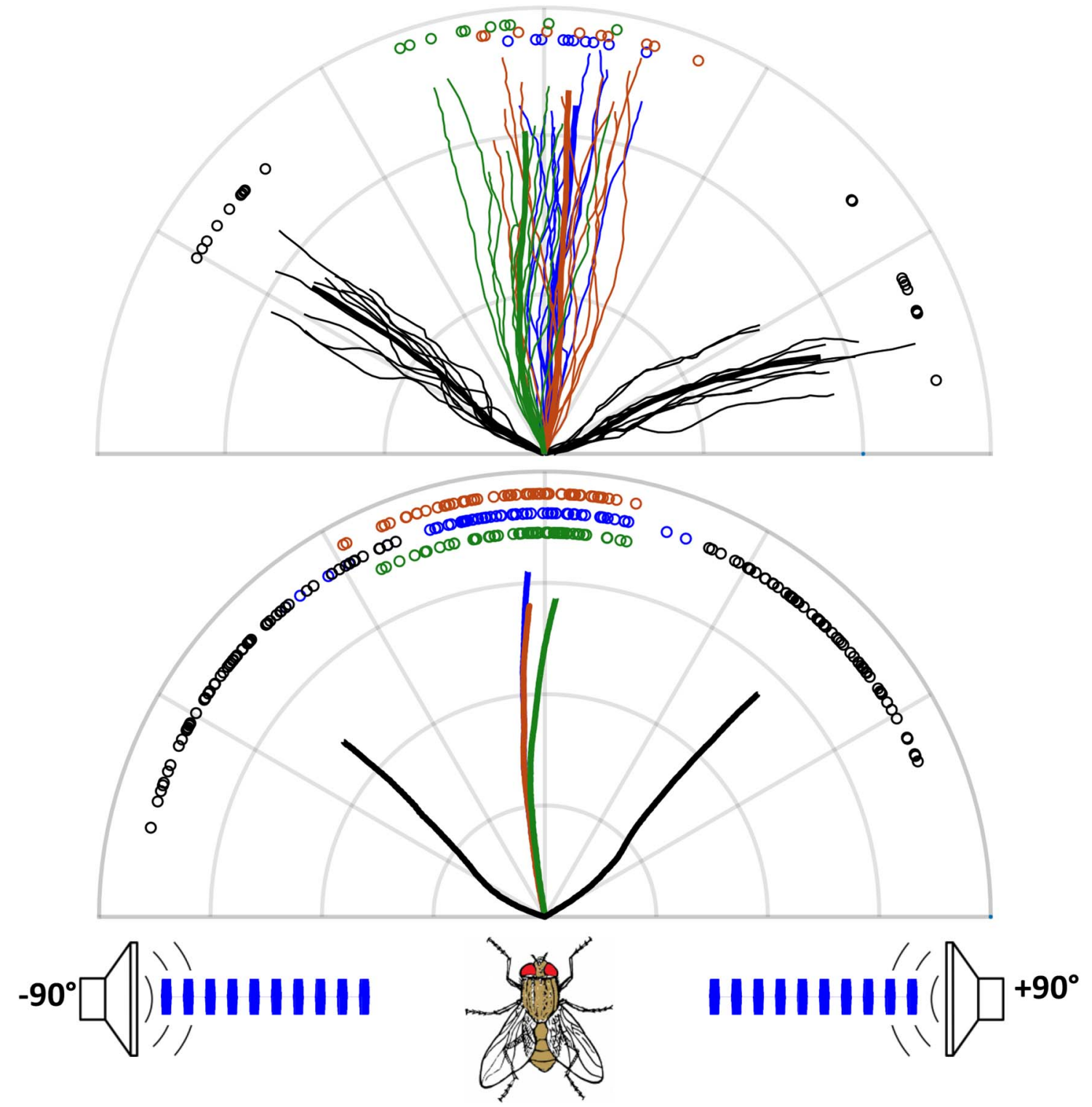

FIGURE 4 | Phonotactic walking responses for standing wave stimuli. The upper panel shows data for a single fly (grid = $5 \mathrm{~cm}$ ). Thick lines represent averages (10 runs per trace); fine lines show the corresponding individual responses. Symbols (here and in subsequent figures) show the angles of the individual responses measured at the halfway point of each walking path, and statistical comparisons were based on these angles. The lower panel shows pooled responses for six flies (10 runs per fly in each trace, grid $=2 \mathrm{~cm}$ ). Black traces are responses to stimuli from single speakers on the corresponding side of the fly. The green traces show responses to both speakers broadcasting in-phase stimuli (symmetric tympanal vibration simulates a single source at $0^{\circ}$ ). The blue and red traces show responses to both speakers broadcasting opposite-phase stimuli adjusted to create a standing wave (anti-symmetric vibration, for equal-amplitude but out-of-phase tympanal vibration), with blue and red traces representing opposite-phase standing waves. The flies' responses were not affected by the phase of the standing wave (single fly - Watson's $U=0.093, p>0.1$; pooled data - Watson's $U=0.0416, p>0.1, n=6$ ). Responses to these conditions are similar to a forward source and show no directional response to cycle-by-cycle phase differences in the stimulus waveform as a cue for directionality (single fly - Rao's homogeneity test for vector direction $=1.55916, p>0.4$; pooled data - Friedman chi-squared $=3.0333, \mathrm{df}=2, p>0.2$ ).

identical conditions using the LDV to validate the stimulus conditions. I show the tympanal vibration data first.

\section{Tympanal Responses}

First, I verified the behavior of the system for conventional freefield auditory stimulation. Tympanal vibration in response to a single source located at $90^{\circ}$ was exactly as predicted by the original analyses of the mechanics of the system (Miles et al., 1995; Robert et al., 1996b). Tympanal vibration in responses to an ipsilateral $\left(90^{\circ}\right.$ azimuth) sound source was greater in amplitude by $12 \mathrm{~dB}$ and leading by $70 \mu$ s relative to the source-contralateral tympanum (Figure 3A).

In the standing wave condition, ipsi- and contralateral tympanal vibrations are equal-amplitude and $180^{\circ}$ out of 


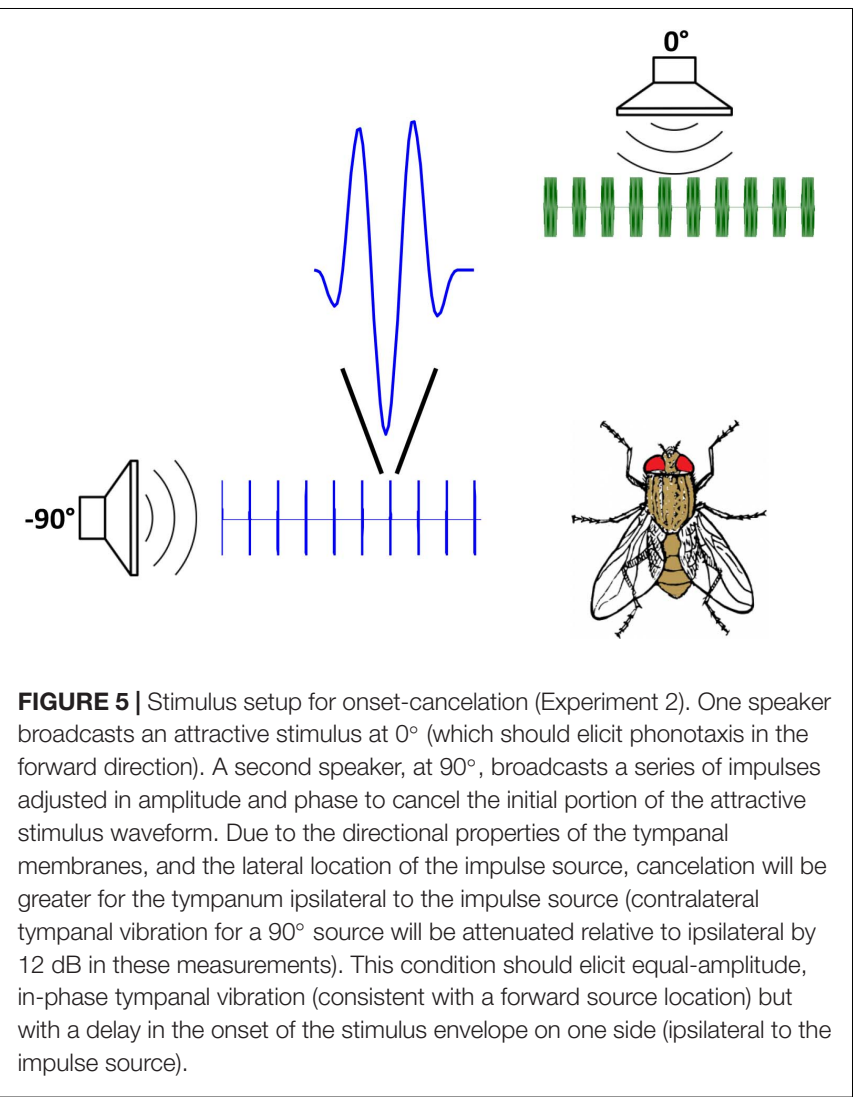

phase, equivalent to a $\pm 100 \mu \mathrm{s}$ tITD (depending on the phase of the standing wave, Figure 3B). In the summed stimulus condition, the tympani showed equal-amplitude, inphase vibration (symmetric mode) similar to a sound source directly ahead $\left(0^{\circ}\right.$ azimuth, data not shown).

\section{Behavioral Responses}

Flies' sound localization behavior was highly consistent within each stimulus condition (Figure 4). In response to stimuli broadcast from either speaker alone, flies showed appropriately oriented phonotaxis. There was no difference in the orientation of phonotaxis between the standing wave and summed stimulus conditions and no effect of a phase reversal in the standing wave. In each condition flies walked directly ahead ( $0^{\circ}$ azimuth). Pure asymmetric mode tympanal vibration did not generate directional cues, despite a $100 \mu \mathrm{s}$ tITD.

Previous work has demonstrated, however, that small differences in the timing of stimulus onset can affect fly responses and mediate selective attention to one among multiple simultaneous sources, via a precedence effect (Lee et al., 2009). The next experiment examined whether time differences in the stimulus amplitude envelope can mediate auditory directionality in response to a single source.

\section{Experiment 2 - Onset Cancelation}

In this experiment, conditions were similar to experiment 1 , except that one speaker was placed at $0^{\circ}$ azimuth (directly forward of the fly), while a second speaker was placed at $90^{\circ}$

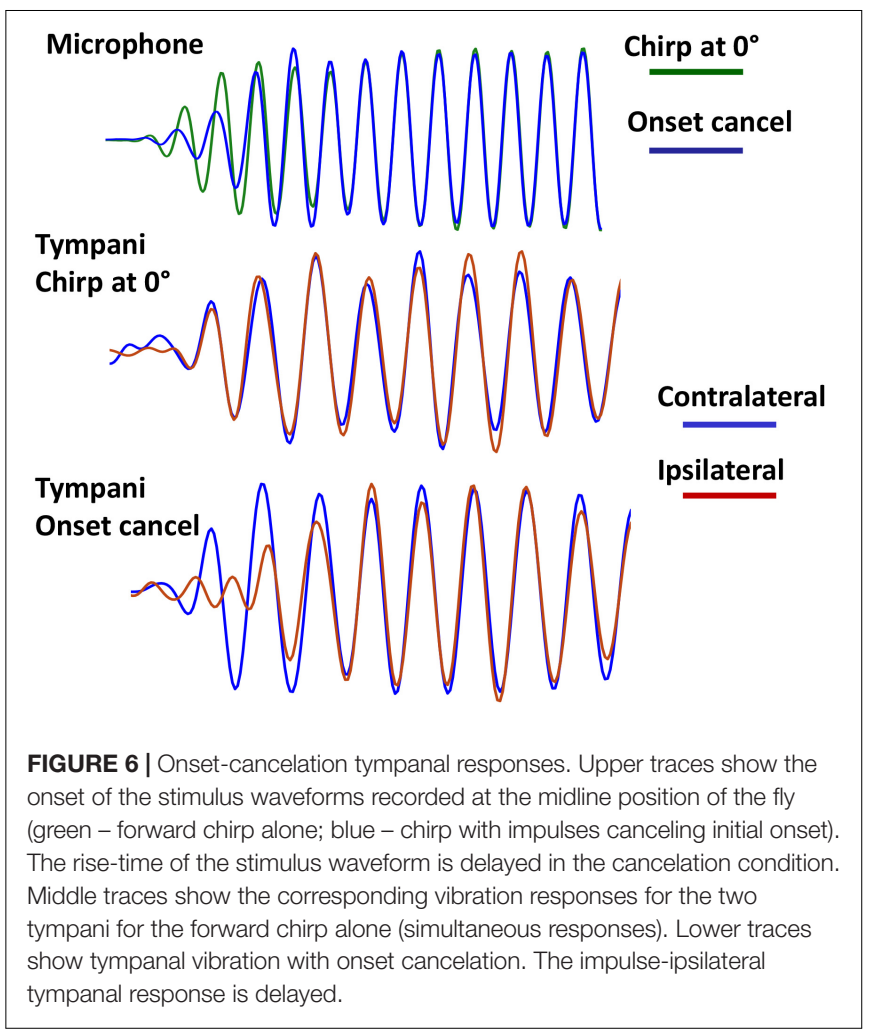

(lateral to the fly). The forward speaker broadcast a synthetic cricket chirp, which should elicit phonotaxis in the forward direction. The second speaker broadcast a train of brief impulses, approximating a half-cycle of the $5 \mathrm{kHz}$ chirp waveform, timed to coincide with the initial onset of the individual pulses of the synthetic chirp, and phase-adjusted to cancel the initial cycle of each chirp pulse (Figure 5). Due to the directional properties of the tympanal membranes, and because the impulse source is located lateral to the fly $\left(90^{\circ}\right.$ azimuth), its effect will be greater on the ipsilateral tympanum than the contralateral by $12 \mathrm{~dB}$ (see above, Figure 3).

The overall result of this stimulus arrangement is that the onset cancelation has a greater effect on the ipsilateral side (relative to the impulse source) than the contralateral, resulting in a delay in the rise-time of the amplitude envelope of the chirp pulses at the ipsilateral tympanum (Figure 6). This has no effect on the overall amplitude of the stimulus at either tympanum but results in a delay in onset timing that is also measurable in summed auditory nerve responses (Figure 7). The additional apparatus required for nerve recordings made it more difficult to calibrate the stimuli in these experiments. Interaural delays measured in auditory nerve responses were variable, with a mean \pm s.d. nITD in the cancelation condition of $48.6 \pm 125.5 \mu \mathrm{s}$ $(n=7)$.

\section{Behavioral Responses}

Fly behavior $(n=5)$ clearly indicated that interaural differences in the timing of stimulus onset constituted a directional cue in the absence of an amplitude difference (Figure 8). Phonotactic 


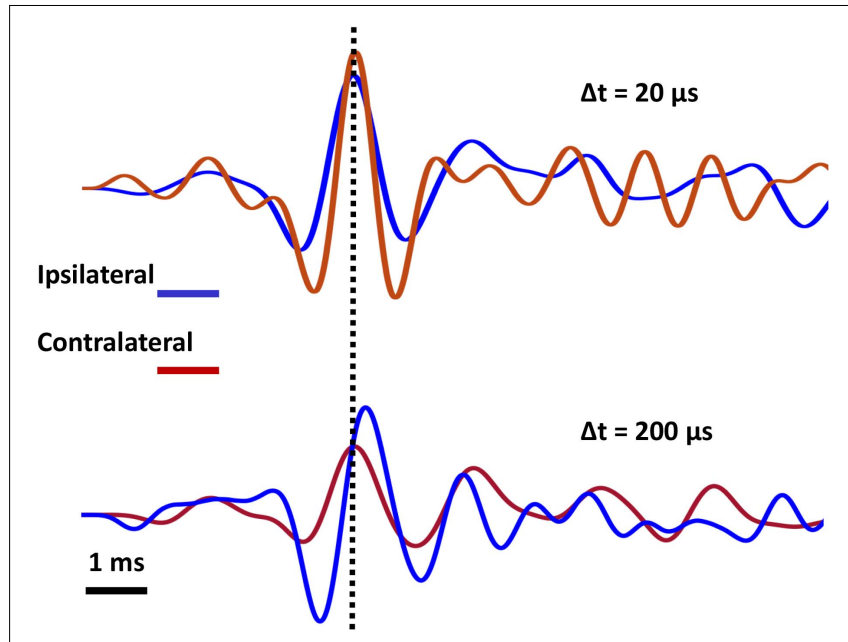

FIGURE 7 | Paired auditory nerve recordings for a stimulus source at $0^{\circ}$ alone (upper traces) and in the onset-cancelation condition (lower traces). Auditory responses are nearly simultaneous for a forward source alone. In the onset-cancelation condition, ipsilateral responses (relative to the cancelation source) are delayed.

walking paths were diverted contralateral to the impulse source (toward the side with leading chirp pulse onsets). Reversing the phase of the impulse waveforms (summing rather than canceling ipsilateral pulse onsets) reversed the effect, and phonotaxis was diverted ipsilateral to the impulse source.

For comparison with free-field auditory directionality, I shifted the position of the attractive sound source to determine what source azimuth elicited responses with similar directionality to the onset-cancelation condition. The directional effect of onset cancelation was equivalent to a source azimuth of $2^{\circ}$ (Figure 9).

\section{DISCUSSION}

Although flies are not sensitive to cycle-by-cycle phase differences in the stimulus waveform, they do show a directional response for stimuli that differ only in the timing of the amplitude envelope, as shown by experiment, and which generate only latency differences in the responses of auditory receptors. However, the magnitude of these directional responses (i.e., the perceived source direction as indicated by the direction of the flies' walking path) is somewhat smaller than would be predicted by measurements of the nITD induced by the stimuli, although still within the range of nITDs elicited by directional sound sources in free field stimulation. Measurements of nITDs in response to variation in sound source azimuth (Mason et al., 2001) showed a slope of $3.5 \mu \mathrm{s} /{ }^{\circ}$. The mean value in these measurements was approximately $50 \mu \mathrm{s}$, which would correspond with an angle of incidence of $\sim 15$ degrees. While these results clearly demonstrated that interaural time differences alone can mediate auditory directionality, they clearly do not rule out a contribution from interaural level differences. Experiments involving dichotic stimulation in orthopteran insects (grasshoppers, katydids, and crickets) have demonstrated a separate contribution of ITD and

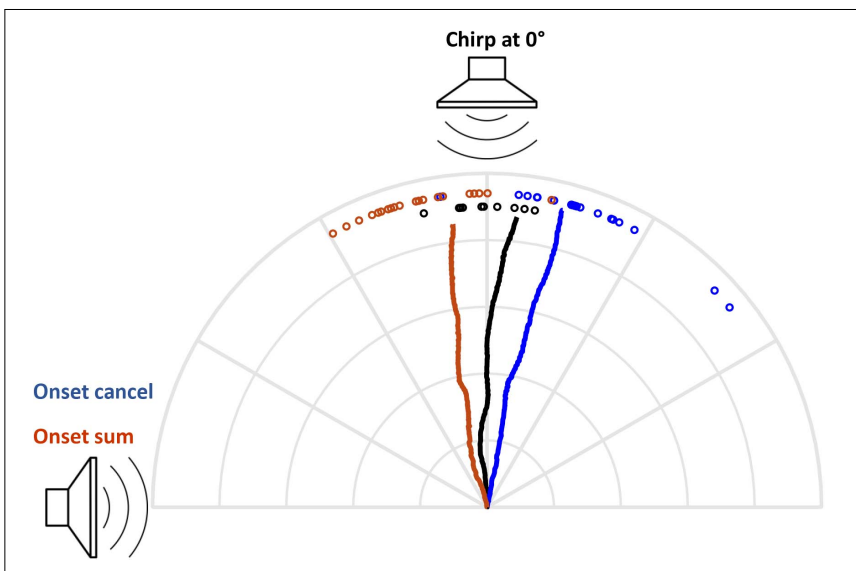

FIGURE 8 | Onset-cancelation behavioral responses for a single fly. Lines represent average $(n=10$ ) walking paths ( $\mathrm{grid}=0.5 \mathrm{~cm}$ ); symbols show the individual response angles (as in Figure 4). Flies walk forward, toward an attractive acoustic stimulus located on midline $\left(0^{\circ}\right.$ azimuth, black trace). When impulses broadcast from a lateral location ( $\pm 90^{\circ}$ azimuth) are timed to cancel the onset of the attractive stimulus pulses, flies' responses are in the direction contralateral to the impulse source (blue line, $0^{\circ}$ alone vs. $0^{\circ}+$ cancel: Watson's $U=0.6626, p<0.001$ ). Reversing the phase of the impulses (so that they sum, rather than cancel the attractive pulse onsets) causes the flies' responses to be oriented toward (ipsilateral to) the impulse source (red line, $0^{\circ}$ alone vs. $0^{\circ}+$ sum: Watson's $\left.U=0.5016, p<0.001\right)$.

ILD cues in directional hearing (Rheinlaender and Mörchen, 1979; Kleindienst et al., 1981; von Helversen and Rheinlaender, 1988; Rheinlaender et al., 2006).

Temporal cues play an integral role in auditory processing (beyond the obvious importance in temporal pattern recognition for the pulsatile acoustic signals of their cricket hosts). Flies' auditory receptors respond almost exclusively to pulse onsets (Oshinsky and Hoy, 2002) and the strength of the response (the fly's perceived stimulus level) is determined by the amplitude increment relative to the noise-floor (effective amplitude, Lee and Mason, 2017). Small time-differences between competing sources mediate selective responses (Lee et al., 2009). While small interaural time-differences (for a single source) mediate directionality.

In a number of functional characteristics, Ormia hearing is convergent with more familiar (i.e., vertebrate) auditory mechanisms, and these may be seen as common principles arising from adaptation to the physics of sound. There are also clear differences, however, which could be consequences of the specific implementation of directionality in Ormia ears. For example, noise can disrupt directional acuity in Ormia and this is not alleviated by spatial separation of noise and signal (flies show no spatial release from masking, Blauert, 1997). Instead, under some circumstances, separation of signal and noise sources increases directional masking in Ormia (Lee and Mason, 2017).

What we know about the flies' hearing suggests that they accomplish as much as possible via peripheral filtering, with their auditory system functioning as a high-resolution, rapidly responding, symmetry detector that makes discrete measurements corresponding to the onset of each pulse in the 


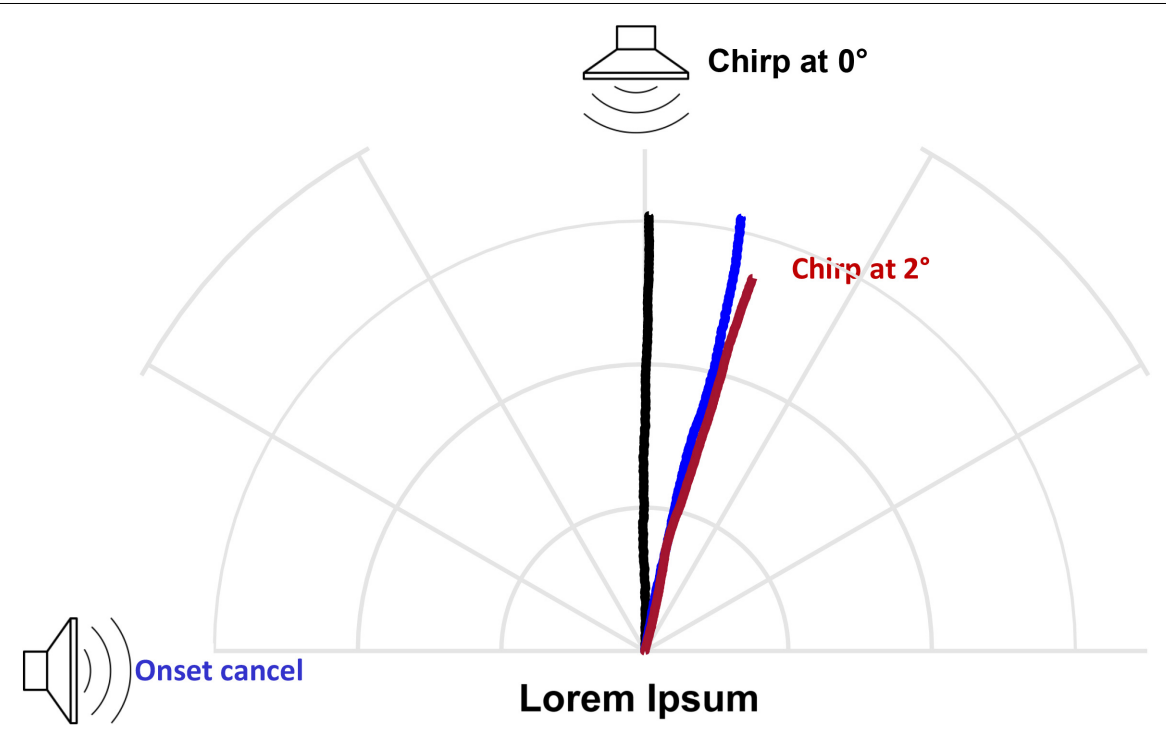

FIGURE 9 | Comparison with free-field auditory directionality. The directional effect of onset cancelation corresponded to a source azimuth of $2^{\circ}$. Chirp at $0^{\circ}$ vs. onset cancel: $n=5$ flies, 10 runs/fly/angle, Watson's $U=0.4607, p<0.001$. Grid $=1 \mathrm{~cm}$.

signal. Flies simply orient to the direction that balances auditory input in time and amplitude. The lack of spatial release from masking and biased response to noise is a consequence of this (Lee and Mason, 2017) and even the precedence effect that mediates source segregation is based on the refractoriness of peripheral receptors (Oshinsky and Hoy, 2002; Lee et al., 2009). This simplified set of information (relative to vertebrate hearing systems) allows for the major functions of hearing: segregation, recognition, and localization of sources, albeit for a specific pre-determined set of stimuli (host communication signals).

The major evolutionary innovation for Ormia hearing is that tympanal coupling relieves them from size limitation in auditory directionality. Comparative and phylogenetic studies (Edgecomb et al., 1995, Robert et al., 1996a) have identified the suite of morphological adaptations that constitute the tympanal ear of this group, and the homologous structures in atympanate flies. Hearing arises as a single-origin evolutionary innovation shared by members of the subfamily Ormiini. The sensory organ itself is derived from a proprioceptive chordotonal organ present, but of uncertain function, in atympanate flies. Surprisingly, tympanal hearing based on the same homologous precursor organ, but independently evolved, was also identified in one species belonging to a second family of flies (Sarcophagidea, Lakes-Harlan et al., 1999). This species shows a number of convergent characteristics with Ormia, including a parasitoid life cycle with an acoustic insect (cicada) as host and directional hearing via coupled eardrums (Robert et al., 1999), although the details of tympanal mechanics are distinct in this species.

Despite the fact that auditory directionality via ICE is now known to be a rather widespread phenomenon (van Hemmen et al., 2016), Ormia should still be considered a highly specialized example, with a number of striking adaptations that appear to optimize their directional acuity despite the relative simplicity (and small scale) of their auditory processing apparatus (both mechanical and neural). An interesting contrast between Ormia and other (vertebrate) examples of ICE is that in most systems, there is a segregation in the frequency domain of the directional cues derived from the coupling mechanism, with ITDs at lower frequencies (relative to the fundamental frequency of the tympanum) and ILDs at higher frequencies (Vedurmudi et al., 2016b), whereas these cues are combined in Ormia (Robert et al., 1996b). On the other hand, the auditory system in Ormia is adapted to exploit the specific temporal and spectral structure of the host cricket acoustic signals; working in a relatively narrow frequency band and detecting the onset timing of individual sound pulses in the trill like call of the host. Expanding the usable bandwidth of systems designed to mimic Ormia hearing has been a major focus for biomimetic engineering efforts inspired by Ormia (Zhang et al., 2018). The subfamily Ormiini, though not a large group, includes nearly 70 species (Lehmann, 2003), with different species exploiting hosts with diverse acoustic signals in terms of both frequency and temporal characteristics. Comparative studies examining how auditory directional mechanisms are adapted to this diversity of signal parameters should be of great interest.

\section{DATA AVAILABILITY STATEMENT}

The raw data supporting the conclusions of this article will be made available by the authors, without undue reservation.

\section{AUTHOR CONTRIBUTIONS}

The author confirms being the sole contributor of this work and has approved it for publication. 


\section{FUNDING}

This work was supported by funding from the Natural Sciences and Engineering Research Council (NSERC) of Canada, (grant numbers 238882 and 241419).

\section{REFERENCES}

Akçakaya, M., and Nehorai, A. (2008). Performance analysis of the Ormia ochracea's coupled ears. J. Acoust. Soc. Am. 124, 2100-2105. doi: 10.1121/1. 2967862

Allen, G. R. (1995). The biology of the phonotactic parasitoid, Homotrixa sp. (Diptera: tachinidae), and its impact on the survival of male Sciarasaga quadrata (Orthoptera: tettigoniidae) in the field. Ecol. Entomol. 20, 103-110. doi: 10. 1111/j.1365-2311.1995.tb00435.x

Blauert, J. (1997). Spatial Hearing: The Psychophysics Of Human Sound Localization. Cambridge, Mass: MIT Press. doi: 10.7551/mitpress/6391.001. 0001

Cade, W. (1975). Acoustically Orienting Parasitoids: fly Phonotaxis to Cricket Song. Science 190, 1312-1313. doi: 10.1126/science.190.4221. 1312

Edgecomb, R., Robert, D., Read, M., and Hoy, R. (1995). The tympanal hearing organ of a fly: phylogenetic analysis of its morphological origins. Cell Tissue Res. 282, 251-268. doi: 10.1007/BF00319116

Gray, D. A., Banuelos, C., Walker, S. E., Cade, W. H., and Zuk, M. (2007). Behavioural specialization among populations of the acoustically orienting parasitoid fly Ormia ochracea utilizing different cricket species as hosts. Anim. Behav. 73, 99-104. doi: 10.1016/j.anbehav.2006. 07.005

Grüner, P., Chaloun, T., and Waldschmidt, C. (2019). A Generalized Model for Two-Element Biomimetic Antenna Arrays. IEEE Trans. Antennas Propag. 67, 1630-1639. doi: 10.1109/TAP.2018.288 8829

Ishfaque, A., and Kim, B. (2018). Fly Ormia Ochracea Inspired MEMS Directional Microphone: a Review. IEEE Sensors J. 18, 1778-1789. doi: 10.1109/JSEN.2017. 2787862

Kleindienst, H.-U., Koch, U. T., and Wohlers, D. W. (1981). Analysis of the cricket auditory system by acoustic stimulation using a closed sound field. J. Comp. Physiol. 141, 283-296. doi: 10.1007/BF006 09930

Kuhn, G. F. (1987). "Physical Acoustics and Measurements Pertaining to Directional Hearing," in In Directional Hearing, eds W. A. Yost and G. Gourevitch (New York, NY: Springer US), 3-25. doi: 10.1007/978-1-46124738-8_1

Lakes-Harlan, R., Stolting, H., and Stumpner, A. (1999). Convergent Evolution of Insect Hearing Organs from a Preadaptive Structure. Proc. Biol. Sci. 266, 1161-1167. doi: 10.1098/rspb.1999.0758

Lee, N., Elias, D. O., and Mason, A. C. (2009). A precedence effect resolves phantom sound source illusions in the parasitoid fly Ormia ochracea. Proc. Natl. Acad. Sci. U. S. A. 106, 6357-6362. doi: 10.1073/pnas.08098 86106

Lee, N., and Mason, A. C. (2017). How spatial release from masking may fail to function in a highly directional auditory system. ELife 6:e20731. doi: 10.7554/ eLife.20731.036

Lehmann, G. U. C. (2003). Review of Biogeography, Host Range and Evolution of Acoustic Hunting in Ormiini (Insecta, Diptera, Tachinidae), Parasitoids of Night-calling Bushcrickets and Crickets (Insecta, Orthoptera, Ensifera). Zool. Anz. J. Comp. Zool. 242, 107-120. doi: 10.1078/0044-523100091

Lisiewski, A. P., Liu, H. J., Yu, M., Currano, L., and Gee, D. (2011). Fly-ear inspired micro-sensor for sound source localization in two dimensions. J. Acoust. Soc Am. 129, E166-E171. doi: 10.1121/1.3565473

Mason, A. C., Oshinsky, M. L., and Hoy, R. R. (2001). Hyperacute directional hearing in a microscale auditory system. Nature 410, 686-690. doi: 10.1038/ 35070564

\section{ACKNOWLEDGMENTS}

I would like to thank Tom Adelman for suggesting the standingwave manipulation; Ali Kanji and Jessica Shaikh for help with rearing flies.

Miles, R. N., Robert, D., and Hoy, R. R. (1995). Mechanically coupled ears for directional hearing in the parasitoid fly Ormia ochracea. J. Acoust. Soc. Am. 98, 3059-3070. doi: 10.1121/1.413830

Miles, R. N., Su, Q., Cui, W., Shetye, M., Degertekin, F. L., Bicen, B., et al. (2009). A low-noise differential microphone inspired by the ears of the parasitoid fly Ormia ochracea. J. Acoust. Soc. Am. 125, 2013-2026. doi: 10.1121/1.3082118

Oshinsky, M. L., and Hoy, R. R. (2002). Physiology of the Auditory Afferents in an Acoustic Parasitoid Fly. J. Neurosci. 22, 7254-7263. doi: 10.1523/JNEUROSCI. 22-16-07254.2002

Pollack, G. S., and Mason, A. C. (2014). "Sound localization in Ormia ochracea: implications of distributed receptor-neuron thresholds," In 11th International Congress of Neuroethology, (Sapporo, Japan: University of Bristol).

Rahaman, A., and Kim, B. (2020). Sound source localization by Ormia ochracea inspired low-noise piezoelectric MEMS directional microphone. Sci. Rep. 10:9545. doi: 10.1038/s41598-020-66489-6

Rheinlaender, J., and Mörchen, A. (1979). 'Time-intensity trading' in locust auditory interneurones. Nature 281, 672-674. doi: 10.1038/28 $1672 \mathrm{a} 0$

Rheinlaender, J., Shen, J.-X., and Römer, H. (2006). Auditory lateralization in bushcrickets: a new dichotic paradigm. J. Comp. Physiol. A Neuroetho.l Sens. Neural Behav. Physiol. 192, 389-397. doi: 10.1007/s00359-0050078-1

Robert, D., Edgecomb, R. S., Read, M. P., and Hoy, R. R. (1996a). Tympanal hearing in tachinid flies (Diptera, Tachinidae, Ormiini): the comparative morphology of an innovation. Cell Tissue Res. 284, 435-448. doi: 10.1007/s0044100 50604

Robert, D., Miles, R. N., and Hoy, R. R. (1996b). Directional hearing by mechanical coupling in the parasitoid fly Ormia ochracea. J. Comp. Physiol. A 179, 29-44. doi: $10.1007 / \mathrm{BF} 00193432$

Robert, D., Miles, R. N., and Hoy, R. R. (1998). Tympanal mechanics in the parasitoid fly Ormia ochracea: intertympanal coupling during mechanical vibration. J. Comp. Physiol. A 183, 443-452. doi: 10.1007/s003590050270

Robert, D., Miles, R. N., and Hoy, R. R. (1999). Tympanal hearing in the sarcophagid parasitoid fly Emblemasoma sp.: the biomechanics of directional hearing. J. Exp. Biol. 202, 1865-1876. doi: 10.1242/jeb.202.14.1865

Römer, H., and Schmidt, A. (2016). Directional hearing in insects with internally coupled ears. Biol. Cybern. 110, 247-254. doi: 10.1007/s00422-015-0672-4

Sakaguchi, K. M., and Gray, D. A. (2011). Host song selection by an acoustically orienting parasitoid fly exploiting a multispecies assemblage of cricket hosts. Anim. Behav. 81, 851-858. doi: 10.1016/j.anbehav.2011.01.024

van Hemmen, J. L., Christensen-Dalsgaard, J., Carr, C. E., and Narins, P. M. (2016). Animals and ICE: meaning, origin, and diversity. Biol. Cybern. 110, 237-246. doi: 10.1007/s00422-016-0702-x

Vedurmudi, A. P., Young, B. A., and van Hemmen, J. L. (2016a). Internally coupled ears: mathematical structures and mechanisms underlying ICE. Biol. Cybern. 110, 359-382. doi: 10.1007/s00422-016-0696-4

Vedurmudi, A. P., Goulet, J., Christensen-Dalsgaard, J., Young, B. A., Williams, R., and van Hemmen, J. L. (2016b). How Internally Coupled Ears Generate Temporal and Amplitude Cues for Sound Localization. Phys. Rev. Lett. 116:028101. doi: 10.1103/PhysRevLett.116.028101

von Helversen, D., and Rheinlaender, J. (1988). Interaural intensity and time discrimination in an unrestraint grasshopper: a tentative behavioural approach. J. Comp. Physiol. 162, 333-340. doi: 10.1007/BF00606121

Wagner, W. E., and Basolo, A. L. (2007). Host preferences in a phonotactic parasitoid of field crickets: the relative importance of host song characters. Ecol. Entomol. 32, 478-484. doi: 10.1111/j.1365-2311.2007.00898.x

Wineriter, S. A., and Walker, T. J. (1990). Rearing phonotactic parasitoid flies diptera, tachinidae, ormiini. Ormia-spp. Entomophaga 35, 621-632. doi: 10 . 1007/BF02375096 
Yack, J., and Dawson, J. (2008). "Insect Ears," in The Senses: A Comprehensive Reference, Vol 3, Audition, Peter Dallos and Donata Oertel, eds I. B. Allan, K. Akimichi, G. S. Gordon, and W. Gerald (Academic Press: San Diego), 35-54. doi: 10.1016/B978-012370880-9.00003-7

Zhang, Y., Reid, A., and Windmill, J. (2018). Insect-inspired acoustic microsensors. Curr. Opin. Insect Sci. 30, 33-38. doi: 10.1016/j.cois.2018. 09.002

Zuk, M., Simmons, L. W., and Rotenberry, J. T. (1995). Acoustically-orienting parasitoids in calling and silent males of the field cricket Teleogryllus oceanicus. Ecol. Entomol. 20, 380-383. doi: 10.1111/j.1365-2311.1995.tb00471.x
Conflict of Interest: The author declares that the research was conducted in the absence of any commercial or financial relationships that could be construed as a potential conflict of interest.

Copyright (c) 2021 Mason. This is an open-access article distributed under the terms of the Creative Commons Attribution License (CC BY). The use, distribution or reproduction in other forums is permitted, provided the original author(s) and the copyright owner(s) are credited and that the original publication in this journal is cited, in accordance with accepted academic practice. No use, distribution or reproduction is permitted which does not comply with these terms. 